\title{
A NEW PINCHING THEOREM FOR COMPLETE SELF-SHRINKERS AND ITS GENERALIZATION
}

\author{
LI LEI, HONGWEI XU, AND ZHIYUAN XU
}

\begin{abstract}
In this paper, we firstly verify that if $M$ is a complete self-shrinker with polynomial volume growth in $\mathbb{R}^{n+1}$, and if the squared norm of the second fundamental form of $M$ satisfies $0 \leq|A|^{2}-1 \leq \frac{1}{18}$, then $|A|^{2} \equiv 1$ and $M$ is a round sphere or a cylinder. More generally, let $M$ be a complete $\lambda$-hypersurface with polynomial volume growth in $\mathbb{R}^{n+1}$ with $\lambda \neq 0$. Then we prove that there exists an positive constant $\gamma$, such that if $|\lambda| \leq \gamma$ and the squared norm of the second fundamental form of $M$ satisfies $0 \leq|A|^{2}-\beta_{\lambda} \leq \frac{1}{18}$, then $|A|^{2} \equiv \beta_{\lambda}$, $\lambda>0$ and $M$ is a cylinder. Here $\beta_{\lambda}=\frac{1}{2}\left(2+\lambda^{2}+|\lambda| \sqrt{\lambda^{2}+4}\right)$.
\end{abstract}

\section{INTRODUCTION}

Suppose $X: M \rightarrow \mathbb{R}^{n+1}$ is an isometric immersion. If the position vector $X$ evolves in the direction of the mean curvature vector $\vec{H}$, this yields a solution of mean curvature flow:

$$
\left\{\begin{array}{l}
\frac{\partial}{\partial t} X(x, t)=\vec{H}(x, t), x \in M \\
X(x, 0)=X(x)
\end{array}\right.
$$

An important class of solutions to the above mean curvature flow equations are self-shrinkers [17, which satisfy

$$
H=-X^{N}
$$

where $X^{N}$ is the projection of $X$ on the unit normal vector $\xi$, i.e., $X^{N}=\langle X, \xi\rangle$. We remark that some authors have a factor $\frac{1}{2}$ on the right-hand side of the defining equation for self-shrinkers.

Rigidity problems of self-shrinkers have been studied extensively. As is known, there are close relations between self-shrinkers and minimal submanifolds. But they are quite different on many aspects. We refer the readers to 14 for the rigidity problems of minimal submanifolds. In [1, Abresch-Langer classified all smooth closed self-shrinker curves in $\mathbb{R}^{2}$. In 1990, Huisken 17] proved that the only smooth closed self-shrinkers with nonnegative mean curvature in $\mathbb{R}^{n+1}$ are round spheres for $n \geq 2$. Based on the work due to Huisken [17, 18, Colding-Minicozzi [11] proved that if $M$ is an $n$-dimensional complete self-shrinker with nonnegative mean curvature and polynomial volume growth in $\mathbb{R}^{n+1}$, then $M$ is isometric to either

2000 Mathematics Subject Classification. 54C24; 53C40.

Key words and phrases. Rigidity theorem, the second fundamental form, self-shrinkers, $\lambda$ hypersurfaces.

Research supported by the National Natural Science Foundation of China, Grant Nos. 11531012, 11371315, 11601478; and the China Postdoctoral Science Foundation, Grant No. 2016M590530. 
the hyperplane $\mathbb{R}^{n}$, a round sphere or a cylinder. In [2], Brendle verified that the round sphere is the only compact embedded self-shrinker in $\mathbb{R}^{3}$ of genus zero.

In 2011, Le-Sesum 20] proved that any $n$-dimensional complete self-shrinker with polynomial volume growth in $\mathbb{R}^{n+1}$ whose squared norm of the second fundamental form satisfies $|A|^{2}<1$ must be a hyperplane. Afterwards, Cao-Li [3] generalized this rigidity result to arbitrary codimension and proved that if $M$ is an $n$-dimensional complete self-shrinker with polynomial volume growth in $\mathbb{R}^{n+q}$, and if $|A|^{2} \leq 1$, then $M$ must be one of the generalized cylinders. In 2014, Ding-Xin 13. proved the following rigidity theorem for self-shrinkers in the Euclidean space.

Theorem A. Let $M$ be an n-dimensional complete self-shrinker with polynomial volume growth in $\mathbb{R}^{n+1}$. If the squared norm of the second fundamental form satisfies $0 \leq|A|^{2}-1 \leq \frac{11}{500}$, then $|A|^{2} \equiv 1$ and $M$ is a round sphere or a cylinder.

In [8, Cheng-Wei proved that if $M$ is an $n$-dimensional complete self-shrinker with polynomial volume growth in $\mathbb{R}^{n+1}$, and if $0 \leq|A|^{2}-1 \leq \frac{3}{7}$, where $|A|$ is constant, then $|A|^{2}=1$.

Recently, $\mathrm{Xu}-\mathrm{Xu}$ [37] improve Theorem $\mathrm{A}$ and proved the following rigidity theorem

Theorem B. Let $M$ be an n-dimensional complete self-shrinker with polynomial volume growth in $\mathbb{R}^{n+1}$. If the squared norm of the second fundamental form satisfies $0 \leq|A|^{2}-1 \leq \frac{1}{21}$, then $|A|^{2} \equiv 1$ and $M$ is a round sphere or a cylinder.

In this paper, we firstly prove the following rigidity theorem for self-shrinkers in the Euclidean space.

Theorem 1.1. Let $M$ be an n-dimensional complete self-shrinker with polynomial volume growth in $\mathbb{R}^{n+1}$. If the squared norm of the second fundamental form satisfies $0 \leq|A|^{2}-1 \leq \frac{1}{18}$, then $|A|^{2} \equiv 1$ and $M$ is one of the following cases:

(i) the round sphere $\mathbb{S}^{n}(\sqrt{n})$;

(ii) the cylinder $\mathbb{S}^{k}(\sqrt{k}) \times \mathbb{R}^{n-k}, 1 \leq k \leq n-1$.

More generally, we consider the rigidity of $\lambda$-hypersurfaces. The concept of $\lambda$ hypersurfaces was introduced independently by Cheng-Wei [7 via the weighted volume-preserving mean curvature flow and McGonagle-Ross [25] via isoperimetric type problem in a Gaussian weighted Euclidean space. Precisely, the hypersurfaces of Euclidean space satisfying the following equation are called $\lambda$-hypersurfaces:

$$
H=-X^{N}+\lambda
$$

where $X^{N}$ is the projection of $X$ on the unit normal vector $\xi$ and $\lambda$ is a constant. In recent years, the rigidity of $\lambda$-hypersurfaces has been investigated by several authors [5, 7, 15, 33]. In [15], Guang showed that if $M$ is a $\lambda$-hypersurface with polynomial volume growth in $\mathbb{R}^{n+1}$, and if $|A|^{2} \leq \alpha_{\lambda}$, then $M$ must be one of the generalized cylinders, where $\alpha_{\lambda}=\frac{1}{2}\left(2+\lambda^{2}-|\lambda| \sqrt{\lambda^{2}+4}\right)$. In the second part of this paper, we prove the following second pinching theorem for $\lambda$-hypersurfaces in the Euclidean space.

Theorem 1.2. Let $M$ be an n-dimensional complete $\lambda$-hypersurface with polynomial volume growth in $\mathbb{R}^{n+1}$ with $\lambda \neq 0$. There exists an positive constant $\gamma$, such that if $|\lambda| \leq \gamma$ and the squared norm of the second fundamental form satisfies $0 \leq|A|^{2}-\beta_{\lambda} \leq \frac{1}{18}$, then $|A|^{2} \equiv \beta_{\lambda}, \lambda>0$ and $M$ must be the cylinder $\mathbb{S}\left(\frac{\sqrt{\lambda^{2}+4}-|\lambda|}{2}\right) \times \mathbb{R}^{n-1}$. Here $\beta_{\lambda}=\frac{1}{2}\left(2+\lambda^{2}+|\lambda| \sqrt{\lambda^{2}+4}\right)$. 


\section{RIGIDITY OF SELF-SHRINKERS}

Let $M$ be an $n$-dimensional complete hypersurface in $\mathbb{R}^{n+1}$. We shall make use of the following convention on the range of indices:

$$
1 \leq i, j, k, \ldots \leq n
$$

We choose a local orthonormal frame field $\left\{e_{1}, e_{2}, \ldots, e_{n+1}\right\}$ near a fixed point $x \in$ $M$ over $\mathbb{R}^{n+1}$ such that $\left\{e_{i}\right\}_{i=1}^{n}$ are tangent to $M$ and $e_{n+1}$ equals to the unit normal vector $\xi$. Let $\left\{\omega_{1}, \omega_{2}, \ldots, \omega_{n+1}\right\}$ be the dual frame fields of $\left\{e_{1}, e_{2}, \ldots, e_{n+1}\right\}$. Denote by $R_{i j k l}, A:=\sum_{i, j} h_{i j} \omega_{i} \otimes \omega_{j}, H:=$ Trace $A$ and $S:=$ Trace $A^{2}$ the Riemann curvature tensor, the second fundamental form, the mean curvature and the squared norm of the second fundamental form of $M$, respectively. We denote the first, the second and the third covariant derivatives of the second fundamental form of $M$ by

$$
\begin{gathered}
\nabla A=\sum_{i, j, k} h_{i j k} \omega_{i} \otimes \omega_{j} \otimes \omega_{k}, \\
\nabla^{2} A=\sum_{i, j, k, l} h_{i j k l} \omega_{i} \otimes \omega_{j} \otimes \omega_{k} \otimes \omega_{l}, \\
\nabla^{3} A=\sum_{i, j, k, l, m} h_{i j k l m} \omega_{i} \otimes \omega_{j} \otimes \omega_{k} \otimes \omega_{l} \otimes \omega_{m} .
\end{gathered}
$$

The Gauss and Codazzi equations are given by

$$
\begin{gathered}
R_{i j k l}=h_{i k} h_{j l}-h_{i l} h_{j k}, \\
h_{i j k}=h_{i k j} .
\end{gathered}
$$

We have the Ricci identities on $M$

$$
\begin{gathered}
h_{i j k l}-h_{i j l k}=\sum_{m} h_{i m} R_{m j k l}+\sum_{m} h_{m j} R_{m i k l} \\
h_{i j k l m}-h_{i j k m l}=\sum_{r} h_{r j k} R_{r i l m}+\sum_{r} h_{i r k} R_{r j l m}+\sum_{r} h_{i j r} R_{r k l m} .
\end{gathered}
$$

We choose a local orthonormal frame $\left\{e_{i}\right\}$ such that $h_{i j}=\mu_{i} \delta_{i j}$ at $x$. By the Gauss equation (2.1) and the Ricci identity (2.3), we have

$$
t_{i j}:=h_{i j i j}-h_{j i j i}=\mu_{i} \mu_{j}\left(\mu_{i}-\mu_{j}\right) .
$$

Set $u_{i j k l}=\frac{1}{4}\left(h_{i j k l}+h_{l i j k}+h_{k l i j}+h_{j k l i}\right)$. Then we have

$$
\begin{aligned}
\sum_{i, j, k, l}\left(h_{i j k l}^{2}-u_{i j k l}^{2}\right) & \geq \frac{6}{16} \sum_{i \neq j}\left[\left(h_{i j i j}-h_{j i j i}\right)^{2}+\left(h_{j i j i}-h_{i j i j}\right)^{2}\right] \\
& =\frac{3}{4} G
\end{aligned}
$$

i.e.,

$$
\left|\nabla^{2} A\right|^{2} \geq \frac{3}{4} G
$$

where $G=\sum_{i, j} t_{i j}^{2}=2\left(S f_{4}-f_{3}^{2}\right)$ and $f_{k}=$ Trace $A^{k}=\sum_{i} \mu_{i}^{k}$. In 11], ColdingMinicozzi introduced the linear operator

$$
\mathcal{L}=\Delta-\langle X, \nabla(\cdot)\rangle=e^{\frac{|X|^{2}}{2}} \operatorname{Div}\left(e^{-\frac{|X|^{2}}{2}} \nabla(\cdot)\right)
$$


They showed that $\mathcal{L}$ is self-adjoint respect to the measure $\rho d \mu$, where $\rho=e^{-\frac{|x|^{2}}{2}}$.

Let $M$ be a self-shrinker with polynomial volume growth. By a computation (see [13, 37]), we have following equalities

$$
\begin{gathered}
\mathcal{L}|A|^{2}=2|A|^{2}-2|A|^{4}+2|\nabla A|^{2}, \\
|\nabla S|^{2}=\frac{1}{2} \mathcal{L} S^{2}+2 S^{2}(S-1)-2 S|\nabla A|^{2}, \\
\left|\nabla^{2} A\right|^{2}=\frac{1}{2} \mathcal{L}|\nabla A|^{2}+\left(|A|^{2}-2\right)|\nabla A|^{2}+3\left(B_{1}-2 B_{2}\right)+\frac{3}{2}|\nabla S|^{2}, \\
\int_{M}\left(B_{1}-2 B_{2}\right) \rho d \mu=\int_{M}\left(\frac{1}{2} G-\frac{1}{4}|\nabla S|^{2}\right) \rho d \mu,
\end{gathered}
$$

where $B_{1}=\sum_{i, j, k, l, m} h_{i j k} h_{i j l} h_{k m} h_{m l}$ and $B_{2}=\sum_{i, j, k, l, m} h_{i j k} h_{k l m} h_{i m} h_{j l}$.

Now we are in a position to prove our rigidity theorem for self-shrinkers in the Euclidean space.

Proof of Theorem 1.1. From (2.7), (2.10) and (2.11), we have

$$
\begin{aligned}
& \int_{M}\left(B_{1}-2 B_{2}\right) \rho d \mu \\
= & \int_{M}\left(\frac{1}{2} G-\frac{1}{4}|\nabla S|^{2}\right) \rho d \mu \\
\leq & \int_{M}\left(\frac{2}{3}\left|\nabla^{2} A\right|^{2}-\frac{1}{4}|\nabla S|^{2}\right) \rho d \mu \\
= & \int_{M}\left[\frac{2}{3}(S-2)|\nabla A|^{2}+2\left(B_{1}-2 B_{2}\right)+\frac{3}{4}|\nabla S|^{2}\right] \rho d \mu .
\end{aligned}
$$

This implies that

$$
\int_{M}\left(B_{1}-2 B_{2}\right) \rho d \mu \geq \int_{M}\left[\frac{2}{3}(2-S)|\nabla A|^{2}-\frac{3}{4}|\nabla S|^{2}\right] \rho d \mu .
$$

By Lemma 4.2 in [13] and Young's inequality, for $\sigma>0$, we have

$$
\begin{aligned}
3\left(B_{1}-2 B_{2}\right) & \leq\left(S+C_{1} G^{1 / 3}\right)|\nabla A|^{2} \\
& \leq S|\nabla A|^{2}+\frac{1}{3} C_{1} \sigma^{2} G+\frac{2}{3} C_{1} \sigma^{-1}|\nabla A|^{3},
\end{aligned}
$$

where $C_{1}=\frac{2 \sqrt{6}+3}{\sqrt[3]{21 \sqrt{6}+103 / 2}}$. Notice that

$$
-\int_{M}\langle\nabla|\nabla A|, \nabla S\rangle \rho d \mu=\int_{M}|\nabla A| \mathcal{L} S \rho d \mu .
$$

This together with (2.8) implies

$$
\begin{aligned}
\int_{M}|\nabla A|^{3} \rho d \mu & =\int_{M}\left(\frac{1}{2} \mathcal{L} S-S+S^{2}\right)|\nabla A| \rho d \mu \\
& =\int_{M}\left[\left(S^{2}-S\right)|\nabla A|-\frac{1}{2}\langle\nabla|\nabla A|, \nabla S\rangle\right] \rho d \mu \\
& \leq \int_{M}\left[\left(S^{2}-S\right)|\nabla A|+\epsilon\left|\nabla^{2} A\right|^{2}+\frac{1}{16 \epsilon}|\nabla S|^{2}\right] \rho d \mu
\end{aligned}
$$


A NEW PINCHING THEOREM FOR COMPLETE SELF-SHRINKERS AND ITS GENERALIZATIOß

From (2.10), (2.11), (2.14), (2.16), we have

$$
\begin{aligned}
& 3 \int_{M}\left(B_{1}-2 B_{2}\right) \rho d \mu \\
\leq & \int_{M}\left(S|\nabla A|^{2}+\frac{1}{3} C_{1} \sigma^{2} G+\frac{2}{3} C_{1} \sigma^{-1}|\nabla A|^{3}\right) \rho d \mu \\
\leq & \int_{M}\left(S|\nabla A|^{2}+\frac{1}{3} C_{1} \sigma^{2} G\right) \rho d \mu \\
& +\frac{2}{3} C_{1} \sigma^{-1} \int_{M}\left[\left(S^{2}-S\right)|\nabla A|+\epsilon\left|\nabla^{2} A\right|^{2}+\frac{1}{16 \epsilon}|\nabla S|^{2}\right] \rho d \mu \\
= & \int_{M}\left[S|\nabla A|^{2}+\frac{2}{3} C_{1} \sigma^{2}\left(B B_{1}-2 B_{2}+\frac{1}{4}|\nabla S|^{2}\right)\right] \rho d \mu \\
& +\frac{2}{3} C_{1} \sigma^{-1} \int_{M}\left[\left(S^{2}-S\right)|\nabla A|+\frac{1}{16 \epsilon}|\nabla S|^{2}\right] \rho d \mu \\
& +\frac{2}{3} C_{1} \sigma^{-1} \epsilon \int_{M}\left[(S-2)|\nabla A|^{2}+3\left(B_{1}-2 B_{2}\right)+\frac{3}{2}|\nabla S|^{2}\right] \rho d \mu .
\end{aligned}
$$

Thus, we obtain

$$
\begin{aligned}
& 3 \theta \int_{M}\left(B_{1}-2 B_{2}\right) \rho d \mu \\
\leq & \int_{M}\left[S+\frac{2}{3} C_{1} \sigma^{-1} \epsilon(S-2)\right]|\nabla A|^{2} \rho d \mu \\
+ & \left(\frac{1}{6} C_{1} \sigma^{2}+C_{1} \sigma^{-1} \epsilon+\frac{1}{24 \epsilon} C_{1} \sigma^{-1}\right) \int_{M}|\nabla S|^{2} \rho d \mu \\
+ & \frac{2}{3} C_{1} \sigma^{-1} \int_{M}\left(S^{2}-S\right)|\nabla A| \rho d \mu,
\end{aligned}
$$

where $\theta=1-\left(\frac{2}{9} C_{1} \sigma^{2}+\frac{2}{3} C_{1} \sigma^{-1} \epsilon\right)$. We restrict $\sigma$ and $\epsilon$ such that $\theta \geq 0$.

Combining (2.13) and (2.18), we have

$$
\begin{aligned}
0 \leq & \int_{M}\left[S+\left(\frac{2}{3} C_{1} \sigma^{-1} \epsilon+2 \theta\right)(S-2)\right]|\nabla A|^{2} \rho d \mu \\
& +\left(\frac{1}{6} C_{1} \sigma^{2}+C_{1} \sigma^{-1} \epsilon+\frac{1}{24 \epsilon} C_{1} \sigma^{-1}+\frac{9}{4} \theta\right) \int_{M}|\nabla S|^{2} \rho d \mu \\
& +\frac{2}{3} C_{1} \sigma^{-1} \int_{M}\left(S^{2}-S\right)|\nabla A| \rho d \mu .
\end{aligned}
$$

To simplify the notation, we put

$$
\begin{gathered}
L_{1}:=\frac{2}{3} C_{1} \sigma^{-1} \epsilon+2 \theta \\
L_{2}:=\frac{1}{6} C_{1} \sigma^{2}+C_{1} \sigma^{-1} \epsilon+\frac{1}{24 \epsilon} C_{1} \sigma^{-1}+\frac{9}{4} \theta .
\end{gathered}
$$

Then (2.19) is reduced to

$$
\begin{aligned}
0 \leq & \int_{M}\left[S+L_{1}(S-2)\right]|\nabla A|^{2} \rho d \mu \\
& +L_{2} \int_{M}|\nabla S|^{2} \rho d \mu+\frac{2}{3} C_{1} \sigma^{-1} \int_{M}\left(S^{2}-S\right)|\nabla A| \rho d \mu
\end{aligned}
$$


When $0 \leq S-1 \leq \delta$, we have

$$
\begin{aligned}
\frac{1}{2} \int_{M}|\nabla S|^{2} \rho d \mu & =\int_{M} S(S-1)^{2} \rho d M-\int_{M}(S-1)|\nabla A|^{2} \rho d \mu \\
& \leq \int_{M}(1-S+\delta)|\nabla A|^{2} \rho d \mu .
\end{aligned}
$$

For $\kappa>0$, we have

$$
\begin{aligned}
\int_{M} S(S-1)|\nabla A| \rho d M \leq & 2(1+\delta) \kappa \int_{M} S(S-1) \rho d \mu \\
& +\frac{1}{8(1+\delta) \kappa} \int_{M} S(S-1)|\nabla A|^{2} \rho d \mu \\
\leq & 2(1+\delta) \kappa \int_{M}|\nabla A|^{2} \rho d \mu \\
& +\frac{1}{8 \kappa} \int_{M}(S-1)|\nabla A|^{2} \rho d M .
\end{aligned}
$$

Substituting (2.21) and (2.22) into (2.20), we obtain

$$
\begin{aligned}
0 \leq & \int_{M}\left[\left(1+L_{1}\right)(S-1)+1-L_{1}\right]|\nabla A|^{2} \rho d \mu \\
& +2 L_{2} \int_{M}(1-S+\delta)|\nabla A|^{2} \rho d \mu \\
& +\frac{4}{3} C_{1} \sigma^{-1}(1+\delta) \kappa \int_{M}|\nabla A|^{2} \rho d \mu \\
& +\frac{1}{12 \kappa} C_{1} \sigma^{-1} \int_{M}(S-1)|\nabla A|^{2} \rho d M \\
= & \int_{M}\left(1+L_{1}-2 L_{2}+\frac{1}{12 \kappa} C_{1} \sigma^{-1}\right)(S-1)|\nabla A|^{2} \rho d \mu \\
& +\int_{M}\left[1-L_{1}+\frac{4}{3} C_{1} \sigma^{-1} \kappa+\left(\frac{4}{3} C_{1} \sigma^{-1} \kappa+2 L_{2}\right) \delta\right]|\nabla A|^{2} \rho d \mu .
\end{aligned}
$$

Let $\sigma=0.616, \epsilon=0.0577$ and $\kappa=0.0434$. By a computation, we have

$$
\begin{gathered}
\theta>0,1+L_{1}-2 L_{2}+\frac{1}{12 \kappa} C_{1} \sigma^{-1}<0, \\
1-L_{1}+\frac{4}{3} C_{1} \sigma^{-1} \kappa<-0.452, \\
\frac{4}{3} C_{1} \sigma^{-1} \kappa+2 L_{2}<8.03 .
\end{gathered}
$$

We take $\delta=1 / 18$. Then the coefficients of the integrals in (2.23) are both negative. Therefore, we have $|\nabla A| \equiv 0$ and $S \equiv 1$, i.e., $M$ either the round sphere $\mathbb{S}^{n}(\sqrt{n})$, or the cylinder $\mathbb{S}^{k}(\sqrt{k}) \times \mathbb{R}^{n-k}, 1 \leq k \leq n-1$.

\section{RIGIDITY OF $\lambda$-HYPERSURFACES}

Let $M$ be an $n$-dimensional complete $\lambda$-hypersurface with polynomial volume growth in $\mathbb{R}^{n+1}$. We adopt the same notations as in Section 2, To simplify the computation, we choose local frame $\left\{e_{i}\right\}$, such that $\nabla_{e_{i}} e_{j}=0$ at $p \in M$, i.e., $\bar{\nabla}_{e_{i}} e_{j}=h_{i j} \xi$, and $h_{i j}=\mu_{i} \delta_{i j}$. Then we have

$$
\nabla_{e_{i}} H=-\nabla_{e_{i}}\langle X, \xi\rangle=h_{i k}\left\langle X, e_{k}\right\rangle,
$$


A NEW PINCHING THEOREM FOR COMPLETE SELF-SHRINKERS AND ITS GENERALIZATION

and

$$
\begin{aligned}
\operatorname{Hess} H\left(e_{i}, e_{j}\right) & =-\nabla_{e_{i}} \nabla_{e_{j}}\langle X, \xi\rangle \\
& =h_{i j k}\left\langle X, e_{k}\right\rangle+h_{i j}-(H-\lambda) h_{i k} h_{k j} .
\end{aligned}
$$

Taking $f_{k}=$ Trace $A^{k}=\sum_{i} \mu_{i}^{k}$, we obtain

$$
\begin{aligned}
\mathcal{L}|A|^{2}= & \Delta|A|^{2}-\left\langle X, \nabla|A|^{2}\right\rangle \\
= & 2 \sum_{i, j} h_{i j} \Delta h_{i j}+2|\nabla A|^{2}-2 \sum_{i, j, k} h_{i j} h_{i j k}\left\langle X, e_{k}\right\rangle \\
= & 2\left(\sum_{i, j} h_{i j} \nabla_{e_{i}} \nabla_{e_{j}} H+H \sum_{i, j, k} h_{i j} h_{j k} h_{k i}-|A|^{4}\right) \\
& +2|\nabla A|^{2}-2 \sum_{i, j, k} h_{i j} h_{i j k}\left\langle X, e_{k}\right\rangle \\
= & 2|A|^{2}-2|A|^{4}+2 \lambda f_{3}+2|\nabla A|^{2} .
\end{aligned}
$$

Proof of Theorem 1.2, Putting $F_{\lambda}=|A|^{4}-|A|^{2}-\lambda f_{3}$, we have

$$
\int_{M} F_{\lambda} \rho d M=\int_{M}|\nabla A|^{2} \rho d M
$$

We also have

$$
|\nabla S|^{2}=\frac{1}{2} \mathcal{L} S^{2}+2 S F_{\lambda}-2 S|\nabla A|^{2}
$$

Notice that

$$
F_{\lambda} \geq|A|^{2}\left(|A|^{2}-1-|\lambda| \cdot|A|\right) .
$$

If $|A|^{2} \geq \beta_{\lambda}:=\frac{1}{2}\left(2+\lambda^{2}+|\lambda| \sqrt{\lambda^{2}+4}\right)$, then $F_{\lambda} \geq 0$. Moreover, if $F_{\lambda}=0$, then $|A|^{2}=\beta_{\lambda}$. Denote by $\alpha_{\lambda}=\frac{1}{2}\left(2+\lambda^{2}-|\lambda| \sqrt{\lambda^{2}+4}\right)$. When $\beta_{\lambda} \leq|A|^{2} \leq \beta_{\lambda}+\delta$, we have the following upper bound for $F_{\lambda}$.

$$
\begin{aligned}
F_{\lambda} & \leq|A|^{2}\left(|A|^{2}-1+|\lambda| \cdot|A|\right) \\
& =|A|^{2}\left(|A|+\sqrt{\beta_{\lambda}}\right)\left(|A|+\sqrt{\alpha_{\lambda}}\right)^{-1}\left(|A|^{2}-\beta_{\lambda}+q_{\lambda}\right) \\
& \leq|A|^{2}\left(|A|^{2}-\beta_{\lambda}+q_{\lambda}\right)\left(1+\frac{\sqrt{\beta_{\lambda}}-\sqrt{\alpha_{\lambda}}}{\sqrt{\beta_{\lambda}}+\sqrt{\alpha_{\lambda}}}\right) \\
& \leq|A|^{2}\left(|A|^{2}-\beta_{\lambda}+r_{\lambda}\right),
\end{aligned}
$$

where $q_{\lambda}=|\lambda| \sqrt{\lambda^{2}+4}, r_{\lambda}=q_{\lambda}+\lambda^{2}+\frac{|\lambda| \delta}{\sqrt{\lambda^{2}+4}}$.

For $\left|\nabla^{2} A\right|$ and the integral of $B_{1}-2 B_{2}$, we obtain the following lemma.

Lemma 3.1. If $M$ is a $\lambda$-hypersurface of $\mathbb{R}^{n+1}$, then we have:

(i) $\left|\nabla^{2} A\right|^{2}=\frac{1}{2} \mathcal{L}|\nabla A|^{2}+\left(|A|^{2}-2\right)|\nabla A|^{2}+3\left(B_{1}-2 B_{2}\right)+\frac{3}{2}|\nabla S|^{2}-3 \lambda C$,

(ii) $\int_{M}\left(B_{1}-2 B_{2}\right) \rho d M=\int_{M}\left(\frac{1}{2} G-\frac{1}{4}|\nabla S|^{2}\right) \rho d M$,

where $B_{1}=\sum_{i, j, k, l, m} h_{i j k} h_{i j l} h_{k m} h_{m l}, B_{2}=\sum_{i, j, k, l, m} h_{i j k} h_{k l m} h_{i m} h_{j l}, C=\sum_{i, j, k, l} h_{i j k} h_{i j l} h_{k l}$, $G=\sum_{i, j} t_{i j}^{2}=2\left(S f_{4}-f_{3}^{2}\right)$ and $t_{i j}=h_{i j i j}-h_{j i j i}=\mu_{i} \mu_{j}\left(\mu_{i}-\mu_{j}\right)$. 
Proof. (i) Applying Ricci identities (2.3) and (2.4), we have

$$
\begin{aligned}
\Delta h_{i j k}= & h_{i j k l l}=\left(h_{i j l k}+h_{i r} R_{r j k l}+h_{r j} R_{r i k l}\right)_{l} \\
= & h_{i j l l k}+h_{r j l} R_{r i k l}+h_{i r l} R_{r j k l}+h_{i j r} R_{r l k l}+\left(h_{i r} R_{r j k l}+h_{r j} R_{r i k l}\right)_{l} \\
= & \left(h_{l j l i}+h_{l r} R_{r j i l}+h_{r j} R_{r l i l}\right)_{k}+h_{r j l} R_{r i k l}+h_{i r l} R_{r j k l}+h_{i j r} R_{r l k l} \\
& +h_{i r l} R_{r j k l}+h_{r j l} R_{r i k l}+h_{i r}\left(R_{r j k l}\right)_{l}+h_{r j}\left(R_{r i k l}\right)_{l} \\
= & H_{j i k}+h_{r k l} R_{r j i l}+h_{r j k} R_{r l i l}+2 h_{r j l} R_{r i k l}+2 h_{r i l} R_{r j k l}+h_{r i j} R_{r l k l} \\
& +h_{i r}\left(R_{r j k l}\right)_{l}+h_{r j}\left(R_{r i k l}\right)_{l}+h_{l r}\left(R_{r j i l}\right)_{k}+h_{r j}\left(R_{r l i l}\right)_{k} .
\end{aligned}
$$

It follows from (3.2) that

$$
H_{j i}=h_{j l i}\left\langle X, e_{l}\right\rangle+h_{i j}+(\lambda-H) h_{i l} h_{j l} .
$$

Since $\langle X, \xi\rangle=\lambda-H$, we compute the covariant derivative of $H_{j i}$

$$
\begin{aligned}
H_{j i k}= & h_{j l i k}\left\langle X, e_{l}\right\rangle+h_{j l i}\left\langle e_{k}, e_{l}\right\rangle+h_{j l i}\left\langle X, \bar{\nabla}_{e_{k}} e_{l}\right\rangle+h_{i j k} \\
& -H_{k} h_{i l} h_{j l}+(\lambda-H)\left(h_{i k l} h_{j l}+h_{i l} h_{j k l}\right) \\
= & h_{j l i k}\left\langle X, e_{l}\right\rangle+2 h_{i j k}-H_{k} h_{i l} h_{j l} \\
& +(\lambda-H)\left(h_{i l} h_{j k l}+h_{j l} h_{i k l}+h_{k l} h_{j l i}\right) .
\end{aligned}
$$

Combining (3.8) and (3.10), we have

$$
\begin{aligned}
\Delta h_{i j k}= & h_{j l i k}\left\langle X, e_{l}\right\rangle+2 h_{i j k}-h_{i l} h_{j l} H_{k} \\
& +(\lambda-H)\left(h_{i l} h_{j l k}+h_{j l} h_{i k l}+h_{k l} h_{j l i}\right) \\
& +h_{r k l} R_{r j i l}+h_{r j k} R_{r l i l}+2 h_{r j l} R_{r i k l}+2 h_{r i l} R_{r j k l}+h_{r i j} R_{r l k l} \\
& +h_{i r}\left(R_{r j k l}\right)_{l}+h_{r j}\left(R_{r i k l}\right)_{l}+h_{l r}\left(R_{r j i l}\right)_{k}+h_{r j}\left(R_{r l i l}\right)_{k} .
\end{aligned}
$$

The Gauss equation (2.15) imples

$$
\begin{aligned}
& h_{i j k}\left(h_{r k l} R_{r j i l}+h_{r j k} R_{r l i l}+2 h_{r j l} R_{r i k l}+2 h_{r i l} R_{r j k l}+h_{r i j} R_{r l k l}\right. \\
& \left.+h_{i r}\left(R_{r j k l}\right)_{l}+h_{r j}\left(R_{r i k l}\right)_{l}+h_{l r}\left(R_{r j i l}\right)_{k}+h_{r j}\left(R_{r l i l}\right)_{k}\right) \\
= & h_{i j k}\left(6 h_{r k l} h_{r i} h_{j l}-6 h_{r k l} h_{r l} h_{i j}+3 h_{r i j} h_{r k} h_{l l}-3 h_{r i j} h_{r l} h_{k l}\right. \\
& \left.+3 h_{i r} h_{r k} h_{j l l}-2 h_{i r} h_{j k} h_{r l l}-h_{i j k} h_{r l}^{2}\right) .
\end{aligned}
$$

From (2.3) and (3.1), we have

$$
\begin{aligned}
h_{i j k}\left(h_{i j l k}-h_{i j k l}\right)\left\langle X, e_{l}\right\rangle & =h_{i j k}\left(h_{i r} R_{r j l k}+h_{r j} R_{r i l k}\right)\left\langle X, e_{l}\right\rangle \\
& =2 h_{i j k} h_{i r} h_{j k} H_{r}-2 h_{i j k} h_{i r} h_{r k} H_{j} .
\end{aligned}
$$


A NEW PINCHING THEOREM FOR COMPLETE SELF-SHRINKERS AND ITS GENERALIZATIOM

Substituting (3.12) and (3.13) into (3.11), we obtain

$$
\begin{aligned}
& \frac{1}{2}(\Delta-\langle X, \nabla \cdot\rangle) h_{i j k}^{2}=h_{i j k}\left(\Delta h_{i j k}-h_{i j k l}\left\langle X, e_{l}\right\rangle\right)+h_{i j k l}^{2} \\
= & h_{i j k}\left(h_{i j l k}-h_{i j k l}\right)\left\langle X, e_{l}\right\rangle+2 h_{i j k}^{2}+h_{i j k l}^{2} \\
& +(\lambda-H) h_{i j k}\left(h_{i l} h_{j l k}+h_{j l} h_{i k l}+h_{k l} h_{j l i}\right)-h_{i j k} h_{i l} h_{j l} H_{k} \\
& +h_{i j k}\left(h_{r k l} R_{r j i l}+h_{r j k} R_{r l i l}+2 h_{r j l} R_{r i k l}+2 h_{r i l} R_{r j k l}\right. \\
& \left.+h_{r i j} R_{r l k l}+h_{i r}\left(R_{r j k l}\right)_{l}+h_{r j}\left(R_{r i k l}\right)_{l}+h_{l r}\left(R_{r j i l}\right)_{k}+h_{r j}\left(R_{r l i l}\right)_{k}\right) \\
= & 2 h_{i j k} h_{i r} h_{j k} H_{r}-2 h_{i j k} h_{i r} h_{r k} H_{j}+2 h_{i j k}^{2}+h_{i j k l}^{2} \\
& +3(\lambda-H) h_{i j k} h_{i j l} h_{k l}-h_{i l} h_{j l} H_{k} h_{i j k} \\
& +h_{i j k}\left(6 h_{r k l} h_{r i} h_{j l}-6 h_{r k l} h_{r l} h_{i j}+3 h_{r i j} h_{r k} h_{l l}-3 h_{r i j} h_{r l} h_{k l}+3 h_{i r} h_{r k} h_{j l l}\right. \\
& \left.-2 h_{i r} h_{j k} h_{r l l}-h_{i j k} h_{r l}^{2}\right) \\
= & \left(2-|A|^{2}\right) h_{i j k}^{2}+h_{i j k l}^{2}+h_{i j k}\left(6 h_{i u} h_{j v} h_{u v k}-3 h_{i j u} h_{u v} h_{k v}\right)-\left.\left.\frac{3}{2}|\nabla| A\right|^{2}\right|^{2} \\
(3.14) \quad & +3 \lambda h_{i j k} h_{i j l} h_{k l} .
\end{aligned}
$$

(ii) It follows from the divergence theorem that

$$
\int_{M} \sum_{i, j}\left(f_{3}\right)_{i j} h_{i j} \rho d M=-\int_{M} \sum_{i, j}\left(f_{3}\right)_{i}\left(h_{i j} \rho\right)_{j} d M .
$$

By the condition $H=-X^{N}+\lambda$, we have

$$
\begin{aligned}
\sum_{i, j}\left(h_{i j} \rho\right)_{j} & =\sum_{i, j} h_{j j i} \rho-\sum_{i, j} h_{i j} \rho\left\langle e_{i}, X\right\rangle \\
& =-e_{i}(\langle\xi, X\rangle) \rho+\sum_{i, j}\left\langle\nabla_{e_{j}} \xi, X\right\rangle \rho \\
& =0 .
\end{aligned}
$$

This together with the divergence theorem implies

$$
\begin{aligned}
\int_{M} \sum_{i, j, k} h_{i k} h_{k j} S_{i j} \rho d M & =-\int_{M} \sum_{i, j, k} h_{i k j} h_{k j} S_{i} \rho d M \\
& =-\frac{1}{2} \int_{M}|\nabla S|^{2} \rho d M
\end{aligned}
$$

Applying Ricci identity (2.3), we get

$$
h_{i j i j}-h_{j i j i}=\mu_{i} \mu_{j}\left(\mu_{i}-\mu_{j}\right) .
$$

Thus, we have

$$
\begin{aligned}
\frac{1}{3} \sum_{i, j} h_{i j}\left(f_{3}\right)_{i j} & =\sum_{i, k} h_{i i k k} \mu_{k} \mu_{i}^{2}+2 \sum_{i, j, k} h_{i j k}^{2} \mu_{i} \mu_{k} \\
& =\sum_{i, k}\left[h_{k k i i}+\left(\mu_{i}-\mu_{k}\right) \mu_{i} \mu_{k}\right] \mu_{k} \mu_{i}^{2}+2 B_{2} \\
& =\sum_{i}\left(\frac{S_{i i}}{2}-\sum_{j, k} h_{i j k}^{2}\right) \mu_{i}^{2}+\sum_{i, k} \mu_{i}^{3} \mu_{k}^{2}\left(\mu_{i}-\mu_{k}\right)+2 B_{2} \\
& =\sum_{i, j, k} \frac{h_{i k} h_{k j}}{2} S_{i j}+S f_{4}-f_{3}^{2}-\left(B_{1}-2 B_{2}\right) .
\end{aligned}
$$


Substituting (3.16), (3.17) and (3.19) into (3.15), we obtain

$$
\int_{M}\left(B_{1}-2 B_{2}\right) \rho d M=\int_{M}\left[S f_{4}-f_{3}^{2}-\frac{1}{4}|\nabla S|^{2}\right] \rho d M .
$$

Combining (2.7) and Lemma 3.1 we derive the following inequality.

$$
\begin{aligned}
\int_{M}\left(B_{1}-2 B_{2}\right) \rho d M= & \int_{M}\left(\frac{1}{2} G-\frac{1}{4}|\nabla S|^{2}\right) \rho d M \\
\leq & \frac{2}{3} \int_{M}\left|\nabla^{2} A\right|^{2} \rho d M-\frac{1}{4} \int_{M}|\nabla S|^{2} \rho d M \\
= & \frac{2}{3} \int_{M}(S-2)|\nabla A|^{2} \rho d M+2 \int_{M}\left(B_{1}-2 B_{2}\right) \rho d M \\
& +\frac{3}{4} \int_{M}|\nabla S|^{2} \rho d M-2 \lambda \int_{M} C \rho d M .
\end{aligned}
$$

This implies

$$
\begin{aligned}
\int_{M}\left(B_{1}-2 B_{2}\right) \rho d M \geq & -\frac{2}{3} \int_{M}(S-2)|\nabla A|^{2} \rho d M \\
& -\frac{3}{4} \int_{M}|\nabla S|^{2} \rho d M+2 \lambda \int_{M} C \rho d M .
\end{aligned}
$$

For any $\sigma>0$, using Lemma 4.2 in [13] and Young's inequality, we have

$(3.23) 3\left(B_{1}-2 B_{2}\right) \leq\left(S+C_{1} G^{1 / 3}\right)|\nabla A|^{2} \leq S|\nabla A|^{2}+\frac{1}{3} C_{1} \sigma^{2} G+\frac{2}{3} C_{1} \sigma^{-1}|\nabla A|^{3}$,

where $C_{1}=\frac{2 \sqrt{6}+3}{\sqrt[3]{21 \sqrt{6}+103 / 2}}$. Notice that

$$
-\int_{M} \nabla|\nabla A| \cdot \nabla S \rho d M=\int_{M}|\nabla A| \mathcal{L} S \rho d M .
$$

This together with (3.3) implies

$$
\begin{aligned}
& \int_{M}|\nabla A|^{3} \rho d M \\
= & \int_{M}\left(F_{\lambda}+\frac{1}{2} \mathcal{L}|A|^{2}\right)|\nabla A| \rho d M \\
= & \int_{M} F_{\lambda}|\nabla A| \rho d M-\frac{1}{2} \int_{M} \nabla|\nabla A| \cdot \nabla S \rho d M \\
\leq & \int_{M} F_{\lambda}|\nabla A| \rho d M+\epsilon \int_{M}\left|\nabla^{2} A\right|^{2} \rho d M+\frac{1}{16 \epsilon} \int_{M}|\nabla S|^{2} \rho d M,
\end{aligned}
$$

for arbitrary $\epsilon>0$. We assume that $S$ satisfies the pinching condition $\beta_{\lambda} \leq S \leq$ $\beta_{\lambda}+\delta$. From (3.4) and (3.5), we have

$$
\begin{aligned}
\frac{1}{2} \int_{M}|\nabla S|^{2} \rho d M & =\int_{M}\left(S-\beta_{\lambda}\right) F_{\lambda} \rho d M-\int_{M}\left(S-\beta_{\lambda}\right)|\nabla A|^{2} \rho d M \\
& \leq \int_{M}\left(-S+\beta_{\lambda}+\delta\right)|\nabla A|^{2} \rho d M
\end{aligned}
$$


A NEW PINCHING THEOREM FOR COMPLETE SELF-SHRINKERS AND ITS GENERALIZATIOIN

For any $\kappa>0$, (3.7) implies

$$
\begin{aligned}
\int_{M} F_{\lambda}|\nabla A| \rho d M \leq & 2\left(\beta_{\lambda}+\delta\right) \kappa \int_{M} F_{\lambda} \rho d M \\
& +\frac{1}{8\left(\beta_{\lambda}+\delta\right) \kappa} \int_{M} F_{\lambda}|\nabla A|^{2} \rho d M \\
\leq & 2\left(\beta_{\lambda}+\delta\right) \kappa \int_{M} F_{\lambda} \rho d M \\
& +\frac{1}{8\left(\beta_{\lambda}+\delta\right) \kappa} \int_{M}\left(|A|^{2}-\beta_{\lambda}+r_{\lambda}\right)|A|^{2}|\nabla A|^{2} \rho d M \\
\leq & 2\left(\beta_{\lambda}+\delta\right) \kappa \int_{M}|\nabla A|^{2} \rho d M \\
& +\frac{1}{8 \kappa} \int_{M}\left(|A|^{2}-\beta_{\lambda}+r_{\lambda}\right)|\nabla A|^{2} \rho d M .
\end{aligned}
$$

For $C$, we have the estimate

$$
|C|=\left|\sum_{i, j, k} \mu_{i} h_{i j k}^{2}\right| \leq|A||\nabla A|^{2} .
$$

Combining (3.23), 3.25) and Lemma 3.1, we obtain

$$
\begin{aligned}
& 3 \int_{M}\left(B_{1}-2 B_{2}\right) \rho d M \\
\leq & \int_{M}\left(S|\nabla A|^{2}+\frac{1}{3} C_{1} \sigma^{2} G+\frac{2}{3} C_{1} \sigma^{-1}|\nabla A|^{3}\right) \rho d M \\
\leq & \int_{M} S|\nabla A|^{2} \rho d M+\frac{1}{3} C_{1} \sigma^{2} \int_{M} G \rho d M \\
& +\frac{2}{3} C_{1} \sigma^{-1} \int_{M} F_{\lambda}|\nabla A| \rho d M+\frac{C_{1}}{24 \sigma \epsilon} \int_{M}|\nabla S|^{2} \rho d M \\
& +\frac{2}{3} C_{1} \sigma^{-1} \epsilon \int_{M}\left|\nabla^{2} A\right|^{2} \rho d M \\
= & \int_{M} S|\nabla A|^{2} \rho d M+\frac{2}{3} C_{1} \sigma^{2} \int_{M}\left(B_{1}-2 B_{2}+\frac{1}{4}|\nabla S|^{2}\right) \rho d M \\
& +\frac{2}{3} C_{1} \sigma^{-1} \int_{M} F_{\lambda}|\nabla A| \rho d M+\frac{C_{1}}{24 \sigma \epsilon} \int_{M}|\nabla S|^{2} \rho d M \\
& +\frac{2}{3} C_{1} \sigma^{-1} \epsilon \int_{M}\left[(S-2)|\nabla A|^{2}+3\left(B_{1}-2 B_{2}\right)+\frac{3}{2}|\nabla S|^{2}-3 \lambda C\right] \rho d M .
\end{aligned}
$$

Hence

$$
\begin{aligned}
& 3 \theta \int_{M}\left(B_{1}-2 B_{2}\right) \rho d M \\
\leq & \int_{M}\left[S+\frac{2}{3} C_{1} \sigma^{-1} \epsilon(S-2)\right]|\nabla A|^{2} \rho d M \\
& +\left(\frac{1}{6} C_{1} \sigma^{2}+\frac{C_{1}}{24 \sigma \epsilon}+C_{1} \sigma^{-1} \epsilon\right) \int_{M}|\nabla S|^{2} \rho d M \\
& +\frac{2}{3} C_{1} \sigma^{-1} \int_{M} F_{\lambda}|\nabla A| \rho d M-2 C_{1} \sigma^{-1} \epsilon \lambda \int_{M} C \rho d M,
\end{aligned}
$$


where $\theta=1-\left(\frac{2}{9} C_{1} \sigma^{2}+\frac{2}{3} C_{1} \sigma^{-1} \epsilon\right)$. When $\theta>0$, this together with (3.22) implies

$$
\begin{aligned}
0 \leq & \int_{M}\left[S+L_{1}(S-2)\right]|\nabla A|^{2} \rho d M+L_{2} \int_{M}|\nabla S|^{2} \rho d M \\
& +\frac{2}{3} C_{1} \sigma^{-1} \int_{M} F_{\lambda}|\nabla A| \rho d M-2 \lambda\left(C_{1} \sigma^{-1} \epsilon+3 \theta\right) \int_{M} C \rho d M,
\end{aligned}
$$

where $L_{1}=\frac{2}{3} C_{1} \sigma^{-1} \epsilon+2 \theta, L_{2}=\frac{1}{6} C_{1} \sigma^{2}+C_{1} \sigma^{-1} \epsilon+\frac{1}{24 \epsilon} C_{1} \sigma^{-1}+\frac{9}{4} \theta$. Substituting (3.26), (3.27) and (3.28) into (3.31), we obtain

$$
\begin{aligned}
& 0 \leq \int_{M}\left[S+L_{1}(S-2)\right]|\nabla A|^{2} \rho d M+2 L_{2} \int_{M}\left(-S+\beta_{\lambda}+\delta\right)|\nabla A|^{2} \rho d M \\
&+\frac{4}{3} C_{1} \sigma^{-1}\left(\beta_{\lambda}+\delta\right) \kappa \int_{M}|\nabla A|^{2} \rho d M+\frac{C_{1}}{12 \sigma \kappa} \int_{M}\left(S-\beta_{\lambda}+r_{\lambda}\right)|\nabla A|^{2} \rho d M \\
&+2|\lambda|\left(C_{1} \sigma^{-1} \epsilon+3 \theta\right) \sqrt{\beta_{\lambda}+\delta} \int_{M}|\nabla A|^{2} \rho d M \\
&= \int_{M}\left[\left(1+L_{1}-2 L_{2}+\frac{C_{1}}{12 \sigma \kappa}\right)\left(S-\beta_{\lambda}\right)+\left(1+L_{1}\right) \beta_{\lambda}-2 L_{1}+2 L_{2} \delta\right. \\
&\left.(3.32) \quad+\frac{4}{3} C_{1} \sigma^{-1}\left(\beta_{\lambda}+\delta\right) \kappa+\frac{C_{1}}{12 \sigma \kappa} r_{\lambda}+2|\lambda|\left(C_{1} \sigma^{-1} \epsilon+3 \theta\right) \sqrt{\beta_{\lambda}+\delta}\right]|\nabla A|^{2} \rho d M .
\end{aligned}
$$

Denote by $\eta_{\lambda}=\frac{4}{3 \sigma} C_{1} \tilde{r}_{\lambda} \kappa+\left(1+L_{1}\right) \tilde{r}_{\lambda}+\frac{C_{1}}{12 \sigma \kappa} r_{\lambda}+2|\lambda|\left(C_{1} \sigma^{-1} \epsilon+3 \theta\right) \sqrt{1+\tilde{r}_{\lambda}+\delta}$, $\tilde{r}_{\lambda}=\beta_{\lambda}-1$. Thus, (3.32) is reduced to

$$
\begin{aligned}
0 \leq & \left(1+L_{1}-2 L_{2}+\frac{C_{1}}{12 \sigma \kappa}\right) \int_{M}\left(S-\beta_{\lambda}\right)|\nabla A|^{2} \rho d M \\
& +\left[1-L_{1}+\frac{4}{3 \sigma} C_{1} \kappa+\left(\frac{4}{3 \sigma} C_{1} \kappa+2 L_{2}\right) \delta+\eta_{\lambda}\right] \int_{M}|\nabla A|^{2} \rho d M
\end{aligned}
$$

Let $\sigma=0.616, \epsilon=0.0577, \kappa=0.0434$. By a computation, we have

$$
\begin{gathered}
\theta>0,1+L_{1}-2 L_{2}+\frac{C_{1}}{12 \sigma \kappa}<0, \\
1-L_{1}+\frac{4}{3 \sigma} C_{1} \kappa<-0.452,2 L_{2}+\frac{4}{3 \sigma} C_{1} \kappa<8.03 .
\end{gathered}
$$

Take $\delta=1 / 18$. There exists an positive constant $\gamma$, such that $\eta_{\lambda} \leq 0.005$ when $|\lambda| \leq \gamma$. Then the coefficients of the integral in (3.33) are both negative. Therefore, $|\nabla A| \equiv 0$. By a classification theorem due to Lawson [19, $M$ must be $\mathbb{S}^{k}(r) \times$ $\mathbb{R}^{n-k}, 1 \leq k \leq n$. For $\lambda \neq 0$, the radius $r$ satisfies $\lambda=\frac{k}{r}-r$. Hence,

$$
\begin{gathered}
r=\frac{\sqrt{\lambda^{2}+4 k}-\lambda}{2}, \\
\mu_{1}=\ldots=\mu_{k}=\frac{1}{2 k}\left(\sqrt{\lambda^{2}+4 k}+\lambda\right),
\end{gathered}
$$

where $\mu_{k}$ is the $k$-th principal curvature of $M$.

We consider the following two cases:

(i) for $\lambda>0$, the squared norm of the second fundamental form $M$ satisfies

$$
S_{k}=\sum_{i=1}^{k} \mu_{i}^{2}=\frac{1}{2 k}\left(\lambda^{2}+2 k+|\lambda| \sqrt{\lambda^{2}+4 k}\right) .
$$


Hence, $S_{1}=\beta_{\lambda}$. When $k \geq 2, S_{k}<\beta_{\lambda}$.

(ii) for $\lambda<0$, by a computation, we have

$$
S_{k}=\sum_{i=1}^{k} \mu_{i}^{2}=\frac{1}{2 k}\left(\lambda^{2}+2 k-|\lambda| \sqrt{\lambda^{2}+4 k}\right) .
$$

When $1 \leq k \leq n, S_{k}<\beta_{\lambda}$.

Therefore, $\lambda>0$ and $M$ must be $\mathbb{S}\left(\frac{\sqrt{\lambda^{2}+4}-|\lambda|}{2}\right) \times \mathbb{R}^{n-1}$.

\section{REFERENCES}

[1] U. Abresch and J. Langer, The normalized curve shortening flow and homothetic solutions, J. Differential Geom., 23(1986), 175-196.

[2] S. Brendle, Embedded self-similar shrinkers of genus 0, Ann. of Math., 183(2016), 715-728.

[3] H. D. Cao and H. Z. Li, A gap theorem for self-shrinkers of the mean curvature flow in arbitrary codimension, Calc. Var. Partial Differential Equations, 46(2013), 879-889.

[4] Q. M. Cheng and H. Nakagawa, Totally umbilic hypersurfaces, Hiroshima Math. J., 20(1990), $1-10$.

[5] Q. M. Cheng, S. Ogata and G. X. Wei, Rigidity theorems of $\lambda$-hypersurfaces, Comm. Anal. Geom., 24(2016), 45-58.

[6] Q. M. Cheng and Y. J. Peng, Complete self-shrinkers of the mean curvature flow, Calc. Var. Partial Differential Equations, 52(2015), 497-506.

[7] Q. M. Cheng and G. X. Wei, Complete $\lambda$-hypersurfaces of weighted volume-preserving mean curvature flow. arXiv:1403.3177.

[8] Q. M. Cheng and G. X. Wei, A gap theorem of self-shrinkers, Trans. Amer. Math. Soc., 367(2015), 4895-4915.

[9] S. S. Chern, M. do Carmo and S. Kobayashi, Minimal submanifolds of a sphere with second fundamental form of constant length, Functional Analysis and Related Fields, SpringerVerlag, Berlin, 1970, 59-75.

[10] T. H. Colding, T. Ilmanen, W. P. Minicozzi II and B. White, The round sphere minimizes entropy among closed self-shrinkers, J. Differential Geom., 95(2013), 53-69.

[11] T. H. Colding and W. P. Minicozzi II, Generic mean curvature flow I; generic singularities, Ann. of Math., 175(2012), 755-833.

[12] Q. Ding and Y. L. Xin, On Chern's problem for rigidity of minimal hypersurfaces in the spheres, Adv. Math., 227(2011), 131-145.

[13] Q. Ding and Y. L. Xin, The rigidity theorems of self-shrinkers, Trans. Amer. Math. Soc., 366(2014), 5067-5085.

[14] J. R. Gu, H. W. Xu, Z. Y. Xu and E. T. Zhao, A survey on rigidity problems in geometry and topology of submanifolds, Proceedings of the 6th International Congress of Chinese Mathematicians, Adv. Lect. Math., 37, Higher Education Press \& International Press, Beijing-Boston, 2016, 79-99.

[15] Q. Guang, Gap and rigidity theorems of $\lambda$-hypersurfaces. arXiv:1405.4871v2.

[16] G. Huisken, Flow by mean curvature of convex surfaces into spheres, J. Differential Geom., 22(1984), 237-266.

[17] G. Huisken, Asymptotic behavior for singularities of the mean curvature flow, J. Differential Geom., 31(1990), 285-299.

[18] G. Huisken, Local and global behaviour of hypersurfaces moving by mean curvature, Differential geometry: partial differential equations on manifolds, Proc. Sympos. Pure Math., 54, Amer. Math. Soc., 1993, 175-191.

[19] B. Lawson, Local rigidity theorems for minimal hypersurfaces, Ann. of Math., 89(1969), 187-197.

[20] N. Q. Le and N. Sesum, Blow-up rate of the mean curvature during the mean curvature flow and a gap theorem for self-shrinkers, Comm. Anal. Geom., 19(2011), 1-27.

[21] L. Lei, H. W. Xu and Z. Y. Xu, On Chern's conjecture for minimal hypersurfaces in spheres, arXiv: $1712.01175 \mathrm{v} 1$.

[22] L. Lei, H. W. Xu and Z. Y. Xu, The second pinching theorem on generalized Chern conjecture, in preparation. 
[23] A. M. Li and J. M. Li, An intrinsic rigidity theorem for minimal submanifolds in a sphere, Arch. Math. (Basel), 58(1992), 582-594.

[24] H. Z. Li and Y. Wei, Classification and rigidity of self-shrinkers in the mean curvature flow, J. Math. Soc. Japan, 66(2014), 709-734.

[25] M. McGonagle and J. Ross, The hyperplane is the only stable smooth solution to the isoperimetric problem in gaussian space, Geom Dedicata, 178(2015), 277-296.

[26] C. K. Peng and C. L. Terng, Minimal hypersurfaces of sphere with constant scalar curvature, Ann. of Math. Stud., 103, Princeton Univ. Press, Princeton, NJ, 1983, 177-198.

[27] C. K. Peng and C. L. Terng, The scalar curvature of minimal hypersurfaces in spheres, Math. Ann., 266(1983), 105-113.

[28] J. Simons, Minimal varieties in Riemannian manifolds, Ann. of Math., 88(1968), 62-105.

[29] Y. J. Suh. and H. Y. Yang, The scalar curvature of minimal hypersurfaces in a unit sphere, Comm. Contemp. Math., 9(2007), 183-200.

[30] S. M. Wei and H. W. Xu, Scalar curvature of minimal hypersurfaces in a sphere, Math. Res. Lett., 14(2007), 423-432.

[31] H. W. Xu, Pinching theorems, global pinching theorems and eigenvalues for Riemannian submanifolds, Ph.D. dissertation, Fudan University, 1990.

[32] H. W. Xu, A rigidity theorem for submanifolds with parallel mean curvature in a sphere, Arch. Math.(Basel), 61(1993), 489-496.

[33] H. W. Xu, L. Lei and Z. Y. Xu, The second pinching theorem for complete $\lambda$-hypersurfaces(in Chinese), Sci. Sin. Math., 48(2018), 1-10.

[34] H. W. Xu and L. Tian, A new pinching theorem for closed hypersurfaces with constant mean curvature in $S^{n+1}$, Asian J. Math., 15(2011), 611-630.

[35] H. W. Xu and Z. Y. Xu, The second pinching theorem for hypersurfaces with constant mean curvature in a sphere, Math. Ann., 356(2013), 869-883.

[36] H. W. Xu and Z. Y. Xu, A new characterization of the Clifford torus via scalar curvature pinching, J. Funct. Anal., 267(2014), 3931-3962.

[37] H. W. Xu and Z. Y. Xu, On Chern's conjecture for minimal hypersurfaces and rigidity of self-shrinkers, J. Funct. Anal., 273(2017), 3406-3425.

[38] H. C. Yang and Q. M. Cheng, Chern's conjecture on minimal hypersurfaces, Math. Z., 227(1998), 377-390.

[39] Q. Zhang, The pinching constant of minimal hypersurfaces in the unit spheres, Proc. Amer. Math. Soc., 138(2010), 1833-1841.

Center of Mathematical Sciences, Zhejiang University, Hangzhou 310027, China

E-mail address: lei-li@zju.edu.cn

Center of Mathematical Sciences, Zhejiang University, Hangzhou 310027, China

E-mail address: xuhw@zju.edu.cn

Center of Mathematical Sciences, Zhejiang University, Hangzhou 310027, China

E-mail address: srxwing@zju.edu.cn 